I N S T I T U T O

$\mathrm{DE}$

M E D I C I N A

T R O P I C A L

DE

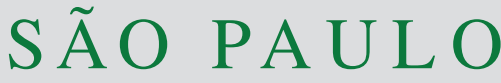

JOURNAL OF THE SÃO PAULO INSTITUTE OF TROPICAL MEDICINE

1 Universidade de São Paulo, Instituto de Medicina Tropical de São Paulo, São Paulo, São Paulo, Brazil

'Universidade de São Paulo, Faculdade de Medicina, Hospital das Clinicas, Instituto do Coração, Laboratório de Genética e Cardiologia Molecular, São Paulo, São Paulo, Brazil

${ }^{3}$ Universidade Federal de Minas Gerais, Departamento de Estatística, Belo Horizonte, Minas Gerais, Brazil

${ }^{4}$ Universidade Federal de São João del-Rey, São João del-Rey, Minas Gerais, Brazil

${ }^{5}$ Universidade Federal de Minas Gerais, Faculdade de Medicina, Departamento de Clínica Médica, Belo Horizonte, Minas Gerais, Brazil

Correspondence to: Lewis F. Buss Universidade de São Paulo, Instituto de Medicina Tropical de São Paulo, Av. Dr. Enéas Carvalho de Aguiar, 470, CEP 05403-000, São Paulo, SP, Brazil

E-mail: lewisbuss@gmail.com

Received: 26 November 2020

Accepted: 22 March 2021

\section{Deriving a parsimonious cardiac endpoint for use in epidemiological studies of Chagas disease: results from the Retrovirus Epidemiology Donor Study-II (REDS-II) cohort}

\author{
Lewis F. Buss ${ }^{\circledR 1}$, Taniela Marli Bes ${ }^{\circledR 1}$, Alexandre Pereira ${ }^{2}$, Larissa Natany ${ }^{3}$, \\ Claudia Di Lorenzo Oliveira ${ }^{(1}$, Antonio Luiz P. Ribeiro ${ }^{5}$, Ester Cerdeira \\ Sabino ${ }^{1}$
}

\section{ABSTRACT}

Chagas cardiomyopathy (ChCM) is a severe consequence of Trypanosoma cruzi infection and has a range of electrocardiographic (ECG) and echocardiographic (ECHO) manifestations. There is a need for a standard and parsimonious research cardiac end point that does not rely on expert panel adjudication, and it is not intended to change the ChCM definition. We use data from the REDS-II cohort to propose a simplified cardiac endpoint. A total of 499 T. cruzi-seropositive blood donors were included. All participants underwent a 12-lead ECG, echocardiogram and clinical examination, and those with abnormal findings were reviewed by a panel of cardiologists who classified cases as having Chagas cardiomyopathy or not. We created an exhaustive set of ECG and ECHO finding combinations and compared these with the panel's classification. We selected the simplest combination that most accurately reproduced the panel's results. Individual ECG and ECHO variables had low sensitivity for panel-defined cardiomyopathy. The best performing combination was right bundle branch block and/or ECHO evidence of left ventricular hypocontractility. This combination had $98 \%$ specificity and $85 \%$ sensitivity for panel-defined ChCM. It was not possible to improve the overall accuracy by addition of any other ECG or ECHO variable. Substituting right bundle branch block for the more inclusive finding of QRS interval > $120 \mathrm{~ms}$ produced similar results. The combination of prolonged QRS interval and/or left ventricular hypocontractility closely reproduced the REDS-II expert panel classification of Chagas ChCM. In conclusion, the simple and reproducible research endpoint proposed here captures most of the spectrum of cardiac abnormalities in Chagas disease.

KEYWORDS: Chagas disease. Trypanosoma cruzi. Cardiomyopathy. Chagas cardiomyopathy.

\section{INTRODUCTION}

Chagas disease (ChD), caused by the protozoan parasite Trypanosoma cruzi, is a neglected tropical disease affecting 6-8 million people worldwide ${ }^{1,2}$. Chagas cardiomyopathy $(\mathrm{ChCM})$ is the most important manifestation ${ }^{3}$. Although a number of electrocardiogram (ECG) and echocardiogram (ECHO) abnormalities are considered typical of $\mathrm{ChCM}^{4}$, these findings are non-specific and occur in other common cardiomyopathies ${ }^{3}$ that are highly prevalent, particularly among older patients with $\mathrm{ChD}^{5}$. For the purpose of research, there is a need for a minimum set of ECG and ECHO findings that can be used as a consistent endpoint to indicate T. cruzi related cardiac damage. 
There are examples of other disease processes in which simplified diagnostic criteria have been derived by comparison of clinical findings with physicians' classification (e.g. Dunkley et al. ${ }^{6}$ ). In a previous cohort, we used clinician adjudication of cardiac status (ChCM or not) on a case-by-case basis ${ }^{7}$. This required blinding to clinical status, a consensus between adjudicators, and a mechanism to resolve conflicting opinions, as well as being expensive and time consuming. Furthermore, while probably the best gold standard available, this approach makes research less reproducible, both across different groups, and at different timepoints in longitudinal studies. Herein, we use data from the well phenotyped REDS-II cohort to derive a parsimonious cardiac endpoint for use in epidemiologic studies of Chagas disease.

\section{MATERIALS AND METHODS}

We present data from the National Heart, Lung, and Blood Institute (NHLBI) Retrovirus Epidemiology Donor Study-II (REDS-II), previously described in detail ${ }^{7,8}$. This cohort is composed of three participant groups. Firstly, a group of $T$. cruzi-seropositive blood donors (SP-BD) identified between 1998 to 2002 in routine serologic screening at the Fundacao Pro-Sangue (Sao Paulo) and Hemominas (Montes Claros) blood centers. SP-BD tested positive on three (ELISA, hemaglutination, and immunofluorescence) or two (ELISA and hemagglutination) serologic assays, with repeat serologic confirmation at cohort enrollment. A negative control group was composed of 488 age- and sex-matched seronegative blood donors (SN-BD) recruited from the same blood banks. A positive control group of 101 patients with established $\mathrm{ChCM}$ was recruited from the specialist outpatient service at the Heart Institute (InCor) - part of the Hospital das Clinicas complex in Sao Paulo, Brazil.

\section{Cohort phenotyping and expert panel review}

The REDS-II cohort includes a wide spectrum of Chagas disease severity as represented across the SP-BD group and the established $\mathrm{ChCM}$ cases. Baseline phenotyping included a standard resting 12-lead ECG, 2-dimentional and tissue Doppler ECHO, as well as clinical history and physical exam. ECG and ECHO results were interpreted in a centralized study unit by investigators blind to clinical and serostatus.

Subjects presenting any alteration on clinical assessment, ECG or ECHO were referred for expert panel review. The panel was composed of three cardiologists experienced in Chagas disease. Patients were classified into four groups: no $\mathrm{ChCM}$, possible, probable or definite ChCM. The following criteria were used to classify a subject as having definite ChCM:

1. Typical findings (reproduced in Table 1) in at least two of the three methods: clinical examination, ECG and $\mathrm{ECHO}$, or;

2. One of the following findings (independent of the other findings): right bundle branch block (RBBB) (with or without left anterior hemi-block [LAHB]) on ECG or apical aneurysm or global left ventricle (LV) hypocontractility on ECHO

However, because none of the above findings are pathognomonic for $\mathrm{ChCM}$, and given the aforementioned complexities in diagnosis, the panel was allowed space for expert subjective judgement and consideration of the global clinical picture, including comorbidities, age, medications etc. Therefore, it is unknown what the relative contribution of each ECG and ECHO finding was to the final classification. We aimed to identify which of the variables available to the panel (Table 1) were most important, and which played only an ancillary role in the final classification. Ultimately, we aimed to identify the most parsimonious set of ECG and ECHO variables that closely reproduced the panel's classification.

\section{Statistical analysis}

As a simplifying assumption, and in line with the original publication ${ }^{7}$, we considered subjects classified as "definite cardiomyopathy" to have ChCM and the other three groups (15 with probable, 53 with possible and 311 cases without) as being free of $\mathrm{ChCM}$. Therefore, there were 120 SP-BD with definite $\mathrm{ChCM}$ and 379 without definite ChCM. An initial descriptive analysis examined each ECG and ECHO finding individually by comparing the prevalence of each across these two groups (with or without definite $\mathrm{ChCM}$ ). As such, the proportion of panelclassified definite ChCM subjects with any given ECG or ECHO finding can be understood as the sensitivity (true positive rate) for that finding.

Conversely, the proportion of subjects without paneldefined $\mathrm{ChCM}$ with a given finding is the false positive rate, or 1 - specificity, associated with that finding.

We then explored different combinations of ECG and ECHO findings and determined their accuracy in reproducing the panel's classification. This analysis was limited to the 499 SP-BDs. We made an exhaustive set of two, three and four variable combinations. For example, a two-variable combination could be ST-T abnormalities on ECG or apical aneurysm or both, such that if a subject 
Table 1 - ECG, echo and clinical findings according to serostatus and expert panel classification in the REDS-II Chagas disease cohort.

\begin{tabular}{|c|c|c|c|c|c|}
\hline \multirow[b]{2}{*}{ ECG and echo findings } & \multicolumn{5}{|c|}{ Clinical groups } \\
\hline & $\begin{array}{c}\text { Seronegative } \\
\text { with CM } \\
n=24\end{array}$ & $\begin{array}{c}\text { Seronegative } \\
\text { BD without } \\
\text { CM } \\
n=464\end{array}$ & $\begin{array}{c}\text { Seropositive } \\
\text { BD without } \\
\text { CM } \\
n=379\end{array}$ & $\begin{array}{c}\text { Seropositive } \\
\text { BD with CM } \\
n=120\end{array}$ & $\begin{array}{c}\text { Patients with } \\
\text { established CM } \\
n=101\end{array}$ \\
\hline \multicolumn{6}{|l|}{ Typical ECG findings, $\mathrm{n}(\%)$} \\
\hline RBBB & $3(12)$ & $0(0)$ & $0(0)$ & $80(67)$ & $41(41)$ \\
\hline Other intraventricular blocks* & $1(4)$ & $2(0)$ & $6(2)$ & $2(2)$ & $7(7)$ \\
\hline Frequent premature vent beats & 2(8) & $3(1)$ & $3(1)$ & $10(8)$ & $13(13)$ \\
\hline Major Q-wave abnormalities & $1(4)$ & $7(2)$ & $4(1)$ & $8(7)$ & $21(21)$ \\
\hline Minor Q/ST-T abnormalities & $0(0)$ & $2(0)$ & $1(0)$ & $3(2)$ & $3(3)$ \\
\hline Major ST-T abnormalities & $2(8)$ & 15(3) & $15(4)$ & $9(8)$ & 19(19) \\
\hline Atrial fibrillation/flutter & $1(4)$ & $0(0)$ & $1(0)$ & 2(2) & $5(5)$ \\
\hline Sinus bradycardia (HR < 40bpm) & $0(0)$ & $0(0)$ & $1(0)$ & $1(1)$ & $0(0)$ \\
\hline $2^{\text {nd }} / 3^{\text {rd }}$ degree AV block & $0(0)$ & $0(0)$ & $0(0)$ & $1(1)$ & $0(0)$ \\
\hline Paced rhythm & $0(0)$ & $0(0)$ & $0(0)$ & $5(4)$ & $20(20)$ \\
\hline \multicolumn{6}{|l|}{$\begin{array}{l}\text { ECG findings possibly related to Chagas, } \\
n(\%)\end{array}$} \\
\hline Sinus bradycardia (40bpm $<\mathrm{HR}<50$ bpm) & $3(12)$ & $23(5)$ & $22(6)$ & $5(4)$ & $7(7)$ \\
\hline Frequent supraventricular premature beats & $1(4)$ & $9(2)$ & $2(1)$ & $7(6)$ & $7(7)$ \\
\hline Left anterior hemiblock & $2(8)$ & $9(2)$ & $18(5)$ & $56(48)$ & $41(41)$ \\
\hline Low QRS voltage & $2(8)$ & $8(2)$ & $13(3)$ & $4(3)$ & $16(16)$ \\
\hline First degree AV block & $1(4)$ & $4(1)$ & $10(2)$ & $11(9)$ & $4(4)$ \\
\hline Minor primary isolated ST/T abnormalities & $4(17)$ & $38(8)$ & $46(12)$ & $13(11)$ & $27(27)$ \\
\hline \multicolumn{6}{|l|}{ Number of ECG findings, $n(\%)$} \\
\hline 0 & $11(46)$ & $366(79)$ & $267(71)$ & $10(8)$ & $2(2)$ \\
\hline 1 & $7(29)$ & $78(17)$ & $88(23)$ & $38(32)$ & $24(24)$ \\
\hline 2 & $4(17)$ & $18(4)$ & $19(5)$ & $44(37)$ & $33(33)$ \\
\hline $3+$ & $2(8)$ & $2(0)$ & $5(1)$ & 28(23) & $42(41)$ \\
\hline \multicolumn{6}{|l|}{ Typical echocardiographic findings, $n(\%)$} \\
\hline Segmental LV contractile abnormalities & 2(9) & $12(3)$ & $11(3)$ & 20(17) & $69(68)$ \\
\hline Apical aneurysm & $0(0)$ & $1(0)$ & $0(0)$ & $1(1)^{\prime}$ & $6(6)$ \\
\hline $\begin{array}{l}\text { Global LV hypocontractility (subjective or } \\
\text { EF }<50 \% \text { ) }\end{array}$ & $11(46)$ & $2(0)$ & $9(2)$ & $33(28)$ & $96(96)$ \\
\hline Left or right ventricular dilation & $4(17)$ & $14(3)$ & $17(4)$ & $25(21)$ & $87(86)$ \\
\hline Intracavity thrombus & $0(0)$ & $0(0)$ & $0(0)$ & $1(1)$ & $2(2)$ \\
\hline \multicolumn{6}{|l|}{ Clinical criteria } \\
\hline Hx of PND & $3(13)$ & $21(5)$ & $35(9)$ & $15(13)$ & $58(58)$ \\
\hline $\mathrm{Hx}$ of Exertional SOB & $5(21)$ & $50(11)$ & 72 (19) & $36(30)$ & $75(74)$ \\
\hline JVP stasis & $0(0)$ & $3(1)$ & $11(3)$ & $5(4)$ & 39 (39) \\
\hline Lower limb edema & $4(17)$ & $20(4)$ & $16(4)$ & $9(8)$ & 39 (39) \\
\hline Lung crepitations & $0(0)$ & $7(2)$ & $5(1)$ & $1(1)$ & $5(5)$ \\
\hline $\mathrm{Hx}$ of faints/LOC & $0(0)$ & $20(4)$ & $31(9)$ & $11(10)$ & $40(40)$ \\
\hline Self-reported palpitations & $6(25)$ & $71(15)$ & $108(28)$ & $40(33)$ & $54(55)$ \\
\hline
\end{tabular}

The classification of ECG and echo findings as "typical" or "possibly related to Chagas" were adapted from Sabino et al. ${ }^{7}$ *left bundle branch block or non-specific intraventricular block. CM = cardiomyopathy; BD = blood donor; ECG = electrocardiogram; $\mathrm{AV}=$ atrioventricular; $\mathrm{HR}=$ heart rate; $\mathrm{LV}=$ left ventricle; $\mathrm{EF}=$ ejection fraction; $\mathrm{Hx}=$ history; $\mathrm{PND}=$ paroxysmal nocturnal dyspnea; SOB = shortness of breath; JVP = jugar venous pressure; LOC = loss of consciousness. Note the percentages in the groups with $\mathrm{CM}$ represents the sensitivity (true positive rate for $\mathrm{CM}$ of that particular finding). In the groups without $\mathrm{CM}$ the percentage represents 1-specificity (false positive rate). Missing values: LAHB 7, first degree AV block 8, segmental LV abnormalities 4, apical aneurysm 5, diastolic dysfunction 6 , intracavity thrombus 4.

had one or both of these findings they would be classified as having ChCM. If they have neither they are classified as being ChCM free. Throughout the document, when referring to variable combinations we use the terminology $\mathrm{X}$ and/or $\mathrm{Y}$ to mean $\mathrm{X}$ or $\mathrm{Y}$ or both, as described above.
As such, by adding variables the proportion of paneldefined ChCM cases would be expected to increase and more false-positives would be expected to be introduced. We used accuracy (true positives + true negatives / total) in order to compare different variable combinations against 
the expert panel, and selected the best performing and most parsimonious combination.

We subsequently evaluated the best performing variable combination by comparing it with the panel's classification for the whole cohort, thus including SN-BD which represent negative controls, and patients with established cardiomyopathy representing positive controls.

\section{Final derivation of proposed cardiac end point}

Using the results of the above analysis, we consulted experienced clinicians in Chagas disease and compared their results with existing guidelines. We determined if the best performing (statistically) cardiac endpoint should be altered on theoretical or pathophysiological grounds, to be more or less inclusive.

\section{Ethics approval and consent to participate}

All participants provided informed consent at the time of cohort enrollment. The study was approved by the Brazilian National Ethics Committee (CONEP N 1312/2006).

\section{RESULTS}

Demographic and clinical characteristics of the entire cohort are presented in Supplementary Table S1. The full set of ECG and ECHO variables considered by the expert panel is shown in Table 1. Focusing on the seropositive blood donor group, all cases in which RBBB was present were considered to have $\mathrm{ChCM}$, and $67 \%$ of seropositive donors with ChCM had this finding. LAHB was the next most prevalent finding among seropositive donors with ChCM: $48 \%$ compared to $5 \%$ among those considered free of ChCM. It is also apparent from Table 1 that the presence of at least one ECG finding identifies $92 \%$ of seropositive donors with ChCM, but would erroneously capture $29 \%$ of the seropositive donors without ChCM. Indeed, 23\% (111/488) of the seronegative donors had at least one ECG alteration, highlighting the lack of specificity inherent in this definition.

Considering the ECHO findings, $28 \%$ of SP-BD with ChCM had global LV hypocontractility (subjective or $\mathrm{EF}<50 \%$ ), whereas only $2 \%$ of those considered not to have $\mathrm{ChCM}$ had this finding. Following this, left or right ventricular dilation was the ECHO variable that appeared to contribute the most to the diagnosis of ChCM.

Clearly no single ECG or ECHO variable serves as an adequate surrogate for the panel's classification. We tested all possible two-variable combinations ( 21 choose $2=210$ combinations) of the ECG and ECHO parameters in Table 1 and calculated the accuracy (true positive + true negatives / total) for each possible two-variable combination (Figure 1A). The best performing combination was RBBB and/or LV hypocontractility, with an accuracy of $95 \%$. Of the 102 SP$\mathrm{BD}$ that were classified as having $\mathrm{ChCM}$ by this definition, 69 had RBBB alone, 22 had LV hypocontractility alone, and
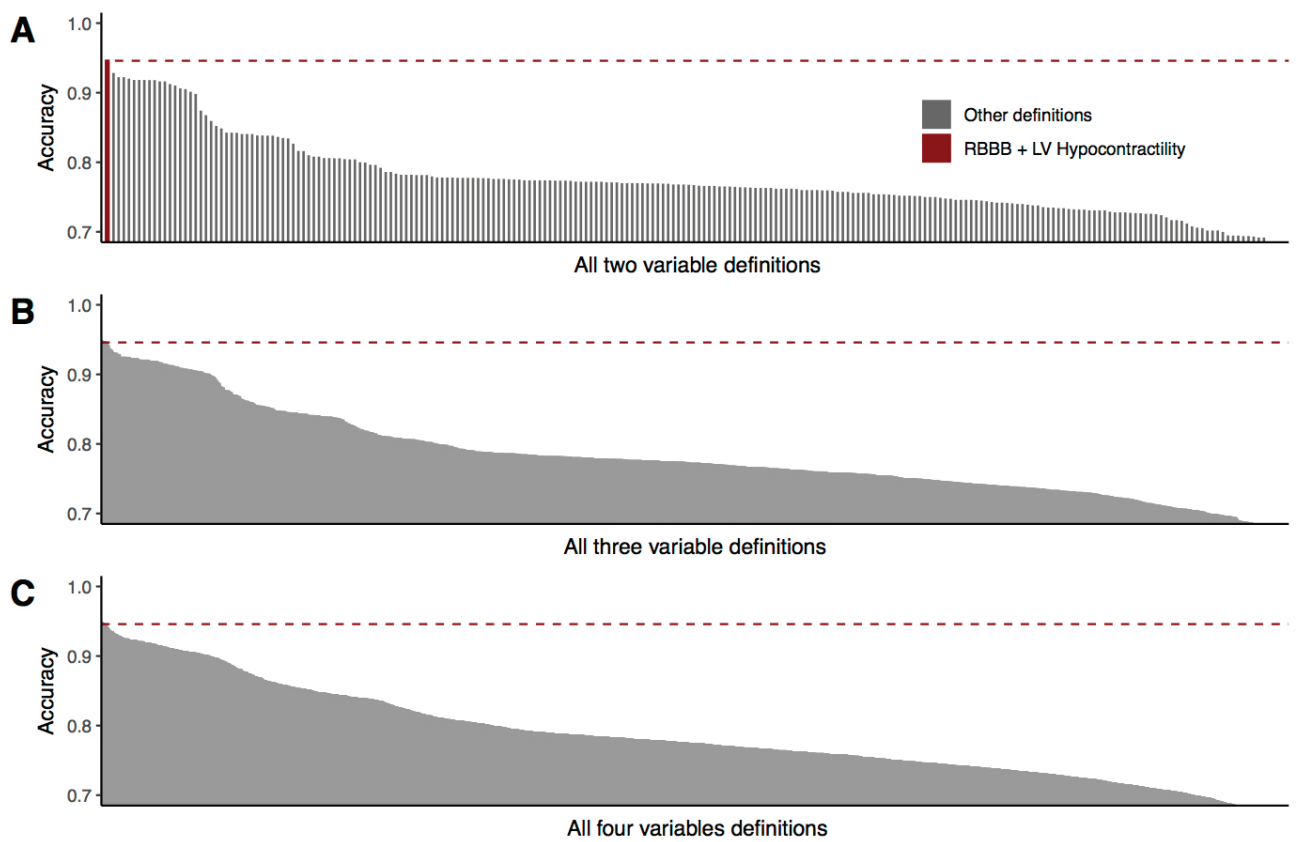

Figure 1 - Accuracy of different ECG and echo parameter combinations to reproduce the expert cardiologist panel classification. The full set of variable combinations was made by selecting all possible ways of choosing 2 (panel A), 3 (panel B) or 4 (panel C) variables from the $21 \mathrm{ECG}$ and echo parameters available to the expert panel. Dashed red line is the accuracy of a two variable combination consisting of RBBB and/or echo evidence of left ventricular hypocontractility (subjective or ejection fraction $<50 \%$ ). 
11 had both findings. We then attempted to improve on this by constructing all possible three-variable combination (21 choose $3=1330$ ) (Figure 1B) and four-variable combination ( 21 choose $4=5985$ ) (Figure 1C). The addition of further variables did not improve the accuracy - i.e. any gain in sensitivity was offset by a reduction in specificity.

Figure 2 shows how the accuracy of the RBBB and/or LV hypocontractility definition varied when applied across the full spectrum of disease. As expected, there is an improved sensitivity ( $92 \%$ vs $85 \%$ ) when it was applied to all subjects (including seronegative donors and patients with established cardiopathy).

The combination of LV hypocontractility and/or RBBB performs well in statistically reproducing the results of the panel's classification. Based on the expert consultation, this end point was considered to be limited from a clinical perspective, as, for example, a patient with isolated left bundle branch block would be classified as free of ChCM. Therefore, we tested the combination of

LV hypocontractility and/or QRS duration $>120 \mathrm{~ms}$, as an alternative to capture all major interventricular blocks. The performance of this combination is shown in Figure 3 and was very similar to the LV hypocontractility and/or RBBB combination.

\section{DISCUSSION}

In this re-analysis of the REDS-II cohort we show that the diagnosis of Chagas cardiomyopathy by a panel of cardiologists was reproduced with 95\% accuracy considering only the presence of QRS prolongation and/or left ventricular hypocontractility. Accuracy was not improved by the addition of other ECG or ECHO variables. This finding could be used to operationalize and standardize a cardiac endpoint in epidemiologic studies of $\mathrm{ChD}$. This would improve comparability across studies, but in particular this approach is more feasible than an expert panel, as clinician adjudication is expensive, timeconsuming and prone to inconsistencies (subjectivity).

There are three classical manifestations of Chagas cardiomyopathy: arrhythmias, cardiac failure and thromboembolic phenomena ${ }^{2,4}$. Arrhythmias arise because Chagas-related fibrosis has a predilection for the conduction system and produce reentry circuits in the ventricular myocardium ${ }^{9}$. Indeed, a wide range of ECG alterations are more prevalent in $T$. cruzi-seropositive patients when compared with seronegative populations ${ }^{10}$. These abnormalities increase with age as a result of the continuous process of cardiac damage over the years ${ }^{5,11}$. In the REDS-II cohort, RBBB was the most discriminatory finding between seropositive and seronegative individuals and this is consistent with other studies in populations with comparable age structure ${ }^{5,10,12,13}$. Other ECG findings, although common in Chagas, are more non-specific, such as left anterior hemi-block, or frequent extra ventricular contractions. It is appropriate, therefore, that RBBB would be a sufficient condition to diagnose Chagas cardiomyopathy in seropositive individuals.

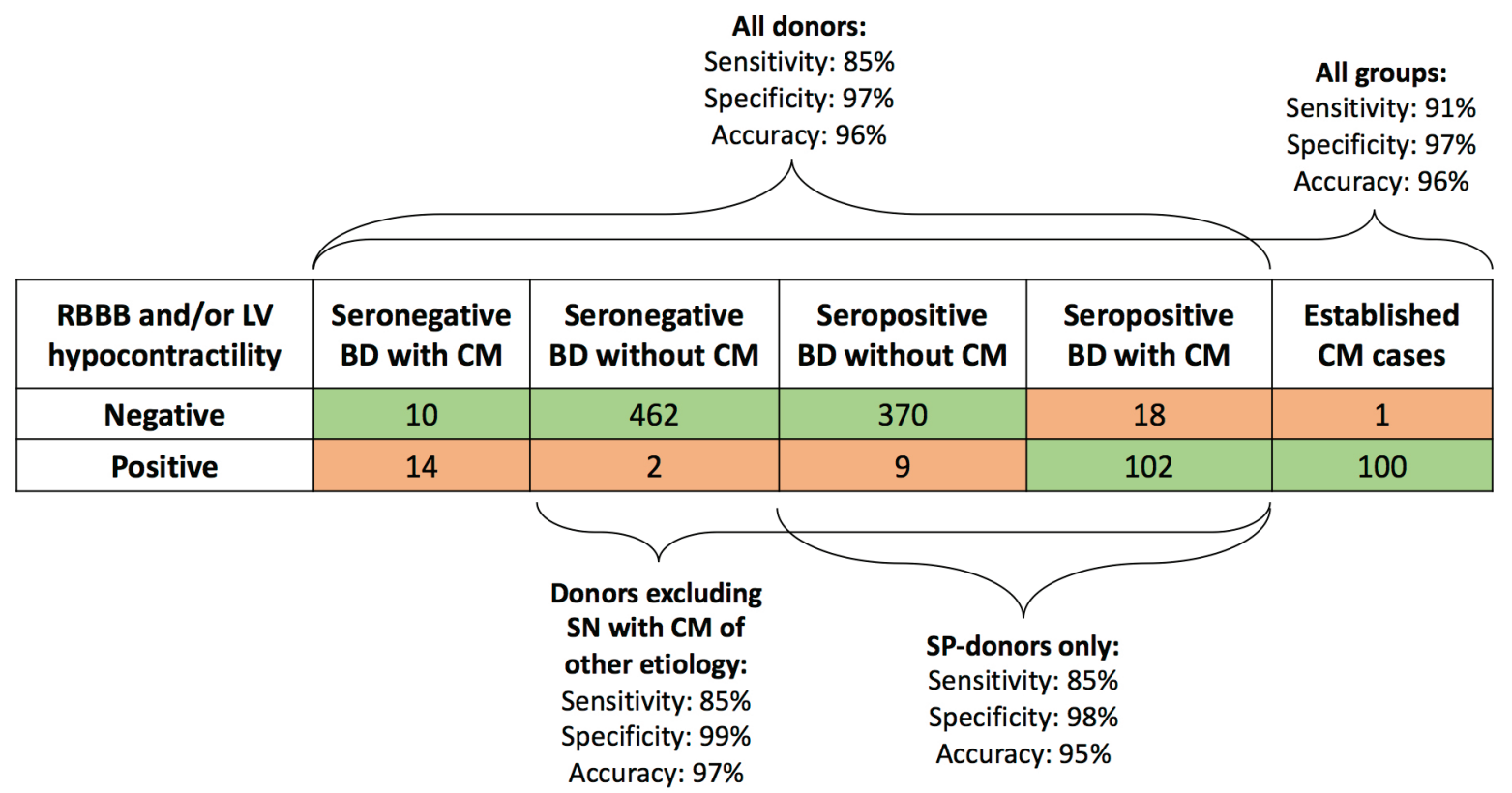

Figure 2 - Sensitivity, specificity and accuracy of right bundle branch block and/or left ventricular hypocontractility to identify ChCM among different clinical groups of the REDS-II cohort. CM = cardiomyopathy; BD = blood donor; RBBB = right bundle branch block; LV = left ventricle; SN = seronegative; SP = seropositive. Among the 102 SP-BD with CM that correctly met the RBBB and/or LV hypocontractility definition, 69 had RBBB alone, 22 had LV hypocontractility alone, and 11 had both findings. 


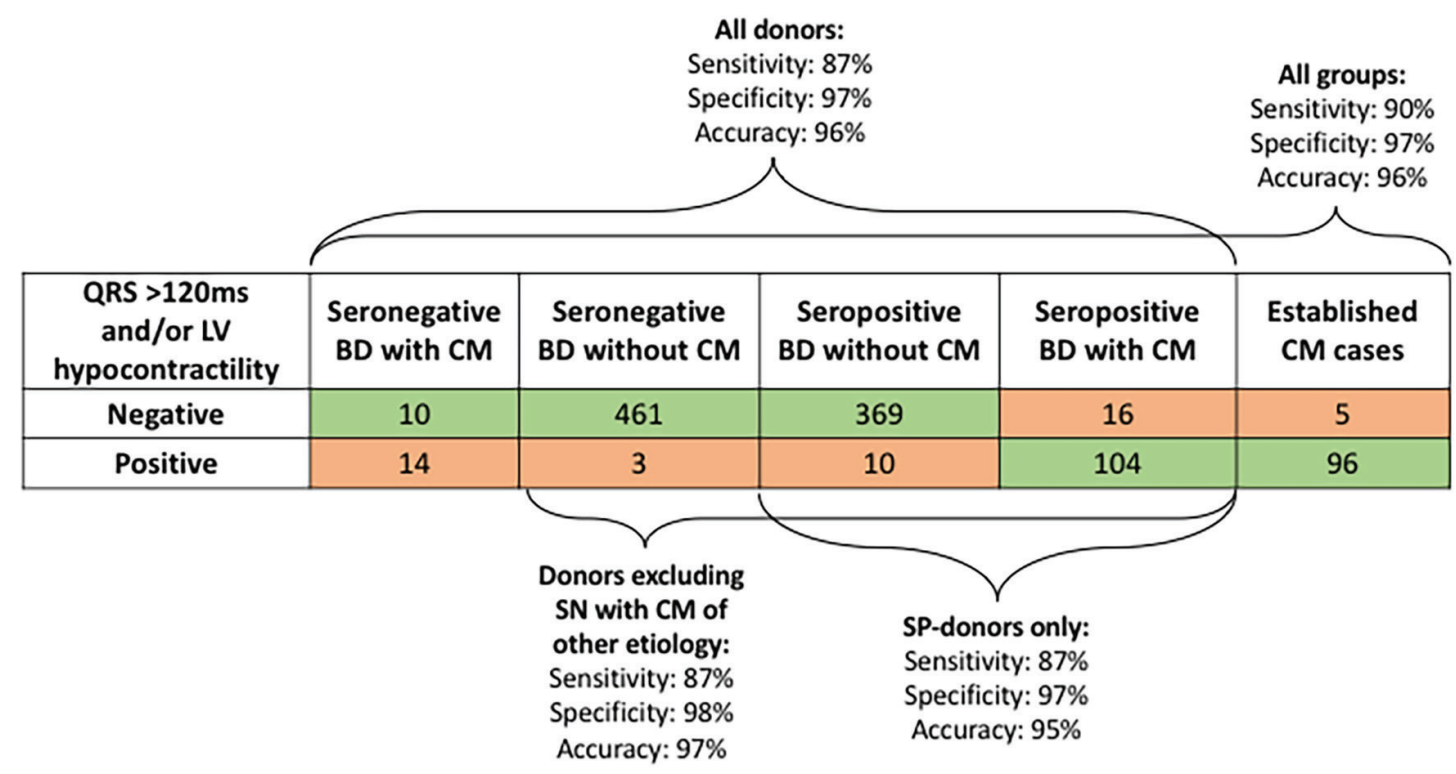

Figure 3 - Sensitivity, specificity and accuracy of QRS duration $>120 \mathrm{~ms}$ and/or left ventricular hypocontractility to identify ChCM among different clinical groups of the REDS-II cohort. CM = cardiomyopathy; $\mathrm{BD}=$ blood donor; $\mathrm{RBBB}=$ right bundle branch block; $\mathrm{LV}=$ left ventricle; $\mathrm{SN}=$ seronegative; $\mathrm{SP}=$ seropositive. Among the $104 \mathrm{SP}-\mathrm{BD}$ with $\mathrm{CM}$ that correctly met the QRS duration $>120 \mathrm{~ms}$ and/ or LV hypocontractility definition, 71 had QRS duration >120ms alone, 22 had LV hypocontractility alone, and 11 had both findings.

There is an important cohort effect among patients with Chagas disease. This has occurred due to effective vector control strategies limiting new infections, and as such most patients enrolled into studies are older adults ${ }^{5,7,14}$ ECG findings that may be typical of Chagas disease in younger patients, such as left anterior hemi-block, have poor specificity in an older age group. This is consistent with our results that ECG alterations other than RBBB played a secondary role in the expert panel's classification.

Addressing the other manifestations of $\mathrm{ChCM}$, heart failure arises in the context of myocardial fibrosis resulting in a dilated cardiomyopathy ${ }^{2,4}$. Both systolic and diastolic impairment are typical ${ }^{15}$. Thromboembolism results from the formation of mural thrombi that occur in the context of extensive cardiac damage. Therefore, it makes sense that a definition that incorporates interventricular conduction abnormalities (the most typical ECG findings) and left ventricular dysfunction would capture most of the spectrum of cardiac manifestations, and therefore perform well as a pragmatic cardiac endpoint for research purposes.

The improvement in sensitivity for ChCM when the definition is applied to the whole cohort (SN-BD, SP-BD, and established ChCM cases) is expected. Sensitivity varies with the spectrum of disease in the population to which the test/diagnostic procedure is being applied ${ }^{16}$. By including the established cases $-96 \%$ of which have left heart failure, and $98 \%$ an abnormal ECG - the sensitivity inevitably increases as the disease is well-established with clear clinical signs. Conversely, as previously reported ${ }^{7}$, $5 \%(24 / 488)$ of seronegative donors were incorrectly classified as having definite $\mathrm{ChCM}$, and half (14/24) met the QRS >120 ms and/or LV failure criterion. This highlights the lack of specificity among ECG and ECHO findings for ChCM. Indeed, a proportion of T. cruzi-infected individuals will have cardiomyopathies of other etiologies that are indistinguishable from ChCM.

Our study has some limitations. Firstly, our results require external validation. Secondly, the end point proposed here requires echocardiography that may not always be available in all settings. Finally, and most fundamentally, we have used the terminology of sensitivity, specificity and accuracy for convenience, but we emphasize that this is not a diagnostic accuracy study per se. We are not presenting an independent comparison between a gold-standard and a potential novel diagnostic procedure. In fact, the ECG and $\mathrm{ECHO}$ variables we evaluated as possible components of a research endpoint, are the same as those used by the expert panel to define ChCM. It is therefore unsurprising that, for example, RBBB has a $100 \%$ specificity among SP-BD, as this ECG finding was a hard criterion for ChCM used by the panel (See Methods and the online appendix of Sabino et $a l^{7}{ }^{7}$ ). As such, our results are correctly interpreted as follows: the diagnosis of definite $\mathrm{ChCM}$ among T. cruziseropositive blood donors, as performed by an expert panel of cardiologists, can be reduced to only two variables prolonged QRS complex and/or LV hypocontractility - with 95\% of subjects remaining classified in the same way.

A prolonged QRS complex or LV failure represents a hard endpoint: subjects meeting this criterion have definite ChCM, but milder/borderline cases may not meet this 
definition. Indeed, the remaining $16(13 \%)$ SP-BD with panel-defined $\mathrm{ChCM}$ that were not identified by our proposed endpoint do not share a single ECG or ECHO finding that is also not present in the panel-defined ChCM-free group. Therefore, these subjects were presumably classified as having ChCM based on a combination of findings that, when considered together, were strongly suggestive of $\mathrm{ChCM}$, but individually were not. Whether the exclusion of this group with normal QRS duration and LV function is epidemiologically important will depend on whether these patients have increased mortality compared to patients with no ECG or ECHO abnormalities. More generally, our proposed cardiac endpoint requires further validation by determining its association with mortality or other important outcomes.

\section{CONCLUSIONS}

The combination of a prolonged QRS complex and/or impaired left ventricular function can serve a simple cardiac endpoint in studies of $\mathrm{ChD}$, avoiding the need for an expert panel adjudication. Future studies should validate this cardiac endpoint in other cohorts and its association with mortality.

\section{AUTHORS' CONTRIBUTIONS}

LFB analyzed the data, interpreted the results, and drafted the manuscript; TB analyzed the data, interpretation the results and drafted the manuscript; AP conceived of the study and interpreted the results; LN curated and analyzed the data; CDO conceived the study, interpretation the results and revised the manuscript; ALPR conceived the study, analyzed the data, interpreted the results, revised the manuscript, and provided supervision; ECS conceived the study, advised on the analysis of the data, interpreted the results, revised the manuscript and provided supervision. All authors read and approved the final version.

\section{FUNDING}

LFB recieves funding from the Fundacao Faculdade de Medicina (FFM) through the Intituto de Medicina Tropical de Sao Paulo of the Universidade de Sao Paulo. This study was supported by the NHLBI, National Institutes of Health, REDS-II International Component (contract HHSN-268200417175-C) and by the Intramural Research Program of the NHLBI. ALPR is supported in part by CNPq (310679/2016-8 and 465518/2014-1) and by FAPEMIG (PPM-00428-17).

\section{REFERENCES}

1. Rassi Jr A, Rassi A, Marin-Neto JA. Chagas disease. Lancet. 2010;375:1388-402.

2. Nunes CP, Beaton A, Acquatella H, Bern C, Bolger AF, Echeverría LE, et al. Chagas cardiomyopathy: an update of current clinical knowledge and management: a scientific statement from the American Heart Association. Circulation. 2018;138:e169-209.

3. Ribeiro AL, Nunes MP, Teixeira MM, Rocha MO. Diagnosis and management of Chagas disease and cardiomyopathy. Nat Rev Cardiol. 2012;9:576-89.

4. Dias CP, Ramos Jr AN, Gontijo ED, Luquetti A, Shikanai-Yasuda MA, Coura JR, et al. $2^{\text {nd }}$ Brazilian Consensus on Chagas Disease, 2015. Rev Soc Bras Med Trop. 2016;49 Suppl 1:360.

5. Ribeiro LP, Marcolino MS, Prineas RJ, Lima-Costa MF. Electrocardiographic Abnormalities in elderly Chagas disease patients: 10-year follow-up of the Bambuí Cohort Study of Aging. J Am Heart Assoc. 2014;3:e00632.

6. Dunkley EJ, Isbister GK, Sibbritt D, Dawson AH, Whyte IM. The Hunter Serotonin Toxicity Criteria: simple and accurate diagnostic decision rules for serotonin toxicity. QJM. 2003;96:635-42.

7. Sabino EC, Ribeiro AL, Salemi MC, Di Lorenzo Oliveira C, Antunes AP, Menezes MM, et al. Ten-year incidence of Chagas cardiomyopathy among asymptomatic Trypanosoma cruziseropositive former blood donors. Circulation. 2013;127:110515.

8. Ribeiro AL, Sabino EC, Marcolino MS, Salemi VM, Ianni BM, Fernandes F, et al. Electrocardiographic abnormalities in Trypanosoma cruzi seropositive and seronegative former blood donors. PLoS Negl Trop Dis. 2013;7:e2078.

9. Barbosa MP, Carmo AA, Rocha MO, Ribeiro LP. Ventricular arrhythmias in Chagas disease. Rev Soc Bras Med Trop. 2015;48:4-10.

10. Rojas LZ, Glisic M, Pletsch-Borba L, Echeverría LE, Bramer WM, Bano A, et al. Electrocardiographic abnormalities in Chagas disease in the general population: a systematic review and meta-analysis. PLoS Neg1 Trop Dis. 2018;12:e0006567.

11. Brito BO, Ribeiro AL. Electrocardiogram in Chagas disease. Rev Soc Bras Med Trop. 2018;51:570-7.

12. Acquatella H, Catalioti F, Gomez-Mancebo JR, Davalos V, Villalobos L. Long-term control of Chagas disease in Venezuela: effects on serologic findings, electrocardiographic abnormalities, and clinical outcome. Circulation. 1987;76:55662.

13. Rassi A Jr, Rassi A, Little WC, Xavier SS, Rassi SG, Rassi AG, et al. Development and validation of a risk score for predicting death in Chagas' heart disease. N Engl J Med. 2006;355:799808 . 
14. Cardoso CS, Ribeiro AL, Oliveira CD, Oliveira LC, Ferreira AM, Bierrenbach AL, et al. Beneficial effects of benznidazole in Chagas disease: NIH SaMi-Trop cohort study. PLoS Negl Trop Dis. 2018;12:e006814.
15. Acquatella H. Echocardiography in Chagas heart disease. Circulation. 2007;115:1124-31.

16. Haynes RB, editor. Clinical epidemiology: how to do clinical practice research. $3^{\text {rd }}$ ed. Philadelphia: Lippincott Williams \& Wilkins; 2006.

\section{SUPPLEMENTARY MATERIAL}

Supplementary Table S1 - Clinical and demographic characteristics of participants in the REDS-II Chagas disease cohort.

\begin{tabular}{|c|c|c|c|c|c|}
\hline \multirow[b]{2}{*}{ ECG and echo findings } & \multicolumn{5}{|c|}{ Clinical groups } \\
\hline & $\begin{array}{l}\text { Seronegative } \\
\text { with CM } \\
n=24 \\
n(\%) \text { or } \\
\text { median(IQR) }\end{array}$ & $\begin{array}{c}\text { Seronegative BD } \\
\text { without } \mathrm{CM} \\
\mathrm{n}=464 \\
\mathrm{n}(\%) \text { or } \\
\text { median(IQR) }\end{array}$ & $\begin{array}{c}\text { Seropositive BD } \\
\text { without } \mathrm{CM} \\
\mathrm{n}=379 \\
\mathrm{n}(\%) \text { or } \\
\text { median(IQR) }\end{array}$ & $\begin{array}{c}\text { Seropositive BD } \\
\text { with CM } \\
\mathrm{n}=120 \\
\mathrm{n}(\%) \text { or } \\
\text { median(IQR) }\end{array}$ & $\begin{array}{c}\text { Patients with } \\
\text { established CM } \\
n=101 \\
n(\%) \text { or } \\
\text { median(IQR) }\end{array}$ \\
\hline Age (years) & $50(44-61)$ & $49(42-58)$ & $49(42-58)$ & $50(44-61)$ & $48(42-54)$ \\
\hline $\begin{array}{l}\text { Sex } \\
\text { Male } \\
\text { Female }\end{array}$ & $\begin{array}{c}15(63) \\
9(37)\end{array}$ & $\begin{array}{l}226(49) \\
238(51)\end{array}$ & $\begin{array}{l}186(49) \\
193(51)\end{array}$ & $\begin{array}{l}75(63) \\
45(38)\end{array}$ & $\begin{array}{l}60(59) \\
41(41)\end{array}$ \\
\hline $\begin{array}{l}\text { Smoking status } \\
\text { Never } \\
\text { Past } \\
\text { Current }\end{array}$ & $\begin{array}{l}12(50) \\
7(29) \\
5(21)\end{array}$ & $\begin{array}{l}243(42) \\
151(32) \\
70(15)\end{array}$ & $\begin{array}{l}222(59) \\
114(30) \\
43(11)\end{array}$ & $\begin{array}{l}61(51) \\
47(39) \\
12(10)\end{array}$ & $\begin{array}{c}47(47) \\
46(46) \\
8(8)\end{array}$ \\
\hline $\begin{array}{l}\text { Comorbidities } \\
\text { Diabetes } \\
\text { Hypertension } \\
\text { Myocardial infarction } \\
\text { Renal disease }\end{array}$ & $\begin{array}{l}2(8) \\
7(29) \\
3(13) \\
1(4)\end{array}$ & $\begin{array}{c}22(5) \\
112(24) \\
2(4) \\
14(3)\end{array}$ & $\begin{array}{c}20(5) \\
89(24) \\
1(3) \\
10(3)\end{array}$ & $\begin{aligned} & 7(6) \\
& 24(20) \\
& 2(2) \\
& 5(4)\end{aligned}$ & $\begin{array}{c}6(6) \\
36(36) \\
12(12) \\
10(10)\end{array}$ \\
\hline $\begin{array}{l}\text { BMI, }\left(\mathrm{kg} / \mathrm{m}^{2}\right) \\
\quad<25 \\
25-29.9 \\
30+\end{array}$ & $\begin{array}{c}5(21) \\
10(42) \\
9(38)\end{array}$ & $\begin{array}{l}127(27) \\
219(47) \\
118(25)\end{array}$ & $\begin{array}{l}127(34) \\
172(45) \\
80(21)\end{array}$ & $\begin{array}{l}44(37) \\
62(52) \\
14(12)\end{array}$ & $\begin{array}{l}44(44) \\
43(43) \\
14(14)\end{array}$ \\
\hline
\end{tabular}

$\mathrm{CM}=$ cardiomyopathy $\mathrm{BD}=$ blood donor BMI = body mass index . 